\title{
Online Healthcare System Using the Concept of Cloud Computing
}

\author{
Prajakta $^{1}$, Prachi $^{2}$, Smita $^{3}$, Prof. P. B. Javalkar ${ }^{4}$ \\ Student, Computer, BSIOTR, Wagholi, India ${ }^{1,2,3}$ \\ Professor, Computer, BSIOTR, Wagholi, India ${ }^{4}$
}

\begin{abstract}
An integrated system to have your health checkups done quickly at best price via nearest path labs and access your reports anywhere anytime on your phone. For health checkups there are many systems available such as thyrocare android app, E-wireless healthcare system, practo etc. So taking in mind these apps were efficient for only specific health related issues. Thyrocare android app is only helpful for thyroid affected persons. E-wireless healthcare system is only helpful for maintaining data about particular hospital. Practo app is helpful for just obtaining the detailed information of doctors in our locality and taking online appointments. Hence to overcome all the drawbacks of the existing systems we are introducing and implementing the online healthcare system using cloud computing concepts which will be efficient for providing online health checkups booking facilities with discounted rates, to get information about preventive measures and tips to avoid contagious diseases, provide different path labs and System will be able to keep track of user's health checkups records, if in case anybody requires the blood then he can broadcast his requirement on the app directly and notification will be send to all the users who are registered having the same blood group via messages and emails. Since, cloud computing is an emerging technology that can be integrated with traditional health management which is used to provide better health services therefore we are using cloud computing concept to develop our system.
\end{abstract}

Keywords: Cloud computing, android application, client portal, MongoDB, MySQL, spring, REST, Apache Tomcat.

\section{INTRODUCTION}

An integrated system to have your health checkups done quickly at best price via nearest path labs and access your reports anywhere anytime on your phone. Based on reports get symptoms and preventive measures on your phone. If in doubts you can contact specialized doctors in your locality from your phone. If you ever need blood its just tap away. Application will broadcast your need to people with same blood group in your locality (city) so that if rare blood groups it will be saviour for you and will save your blood bank expenses.

Now a day's, in fast moving world, its important to keep focus on your health and do regular health checkups to find out heath related issues at early stages. Also it is important to get information about preventive measures and tips to avoid contagious disease. Hence it is today's need to have an integrated system which will have tie ups with different path labs and provide an online health checkup booking facilities with discounted rates. System will be able to keep track of user's health checkup records. The system includes three components: Cloud Computing, Android application, Client Portal.

Cloud is used for creating the virtual environment which having four categories namely Public cloud, Private cloud, Hybrid Cloud and Community cloud. Public cloud is a model in which service provider makes the data publicly available on the internet. Private cloud is a model which is only limited to corporate firewall or we can share the data privately. Hybrid cloud is the combination of both public as well as private cloud. Community cloud is a model which is shared among several organizations or the group of people.
Since, cloud computing is one of the emerging technologies which has an increasing impact on both private and public sectors. But for developing our system i.e. Online Healthcare System using cloud computing concepts we are using public cloud in which the Processors will be shared publicly and users data, reports will be secured because one will get them on his/her email. Cloud computing provides many services which includes Infrastructure as a Service (IaaS), Platform as a Service (PaaS), Software as a Service (SaaS), security as a Service (SecaaS) and Database as a Service (DBaaS)

DBaaS is a service which does not require any expert administrator or any special maintenance to manage the database and its functions. As in our system database plays a vital role, therefore we are using DBaaS to maintain the data at backend. For designing the database we are using the concepts of data modelling languages i.e. MySQL, MongoDB. Database is having the connectivity with server i.e. Apache Tomcat.

For finding the location of the specific user Geolocation API is used which will help to identify residual end points i.e. latitude and longitude of the area. Integrating android application and cloud computing concepts can resolve many health issues and help user to perform many tasks like health tests, blood donation and obtaining preventive measures, symptoms of any disease.

\section{LITERATURE REVIEW}

1.Doukas, C., and Maglogiannis, I.," Managing Wearable Sensor Data through Cloud Computing", Third IEEE International Conference. 
Managing Wearable Sensor Data through Cloud among medical institute. Cloud computing will act as Computing applications and facilities includes patient central repository to implement such application and it monitoring and emergency response. It also introduces will provide the connectivity among the environment to many challenges such as interoperability and availability of heterogeneous resources, data storage and management, unified and ubiquitous access issues. The solution to overcome the above mentioned issues the concept of Cloud Computing concept is considered to be efficient. For developing and presenting many platforms are used such as open hardware and software which collects motion and heartbeat data and further they are stored wirelessly on an open Cloud infrastructure for monitoring and processing. The proposed system may be used to promote the independent living of patient.

2.Ibnualim, I., and Supangkat, S, H. "Design of Health Social Media to Improve the Quality of Patient's Recovery", International conference on Cloud Computing and Social Networking (ICCCSN), pp: 1-4, 2012.

Design of Health Social Media to Improve the Quality of Patient's Recovery helps to create the communication between doctor and patient. The communication between them must be effective in order to satisfy the patient's requirement. Social media acts as a central repository with great impact in socializing activities and also it can be one alternative to solve communication problem. Since the existing social media are not fully in conformation with the needs of the health sector. In this application the research about appropriate design of health social media is carried out. There are three characteristics and functionalities defined of health community which fulfils by health social media. Those characteristics are confidentiality of information and security, the importance of the truth of information, difference of social data and health data, and the credibility of users that involved in health community.

\section{Ratnam , K, A., and Dominic, P, D, D., " Cloud Services} Enhancing the Malaysian Healthcare Sector", international conference on Computer \& Information Science (ICCIS), Vol: 2, pp: 604-608, 2012.

Cloud services - Enhancing the Malaysian hospital sector is the device where application services are installed manually on devices wherever they are required. For example, the software relevant to view MRI images is only available to particular devices which are located at the scanner itself. In order to integrate resource and data sharing for providing better services to patients, the medical world eco-system is using software as service based architecture (SAAS) rather than a device oriented based architecture. This helps in checking the availability of application service on every device. Devices ranges from smart phones to PDA's and workstations are located both locally as well as remotely. To make compatibility with medical related applications in a dynamic environment, medical devices must be treated as a part of the cloud, in which software modules are deployed automatically as and when required. In this we will implement an architecture which will tightly link with the various medical institute at the present, accessing the medical information from the application is not similar

4. Saldarriaga, A, J., Pérez, J.J Restrepo, J., and Bustamante, J." A mobile application for ambulatory electrocardiographic monitoring in clinical and domestic environments", IEEE Health Care Exchanges (PAHCE), 2013 Pan American ,pp: 1-4, 2013.

A mobile application for ambulatory electrographic monitoring in clinical and domestic environments provides computational power and interconnection capacities. It is contributing various Smartphone's and tablets to become an essential tool for the medical sector. Developed countries have proved that mobile devices are useful for tracking, reading updating, monitoring, and treatment assistance; also mobile devices are considered to be portable health care support machines. Here a mobile application for ambulatory electrocardiographic monitoring based on Android and iOS i.e. iPhone Operating System is developed. It also provides accuracy in daily activities connecting different areas. The application is composed of several buttons, switches, sliders and rotating screens having the two scenarios like patient and doctor mode which allows medical personnel to define different configurations. Both these modes permit users to visualize in real time captured readings and tracings. Its benefits have to improve the capabilities and decreased complexities, becoming one of the main instruments at hospital environments. It has been proved that some devices have best performance through the use of cloud computing services which have low power consumption and the low number of connections. This will help to gather medical personnel the use of capacities for mobile devices through medical applications and guide diagnose procedures with efficiency.

\section{MOTIVATION}

Motivation strategies should approach these complex problems holistically: provide opportunities for career development, make efforts to ensure adequate compensations, and promote positive work environments, including supportive supervision. Human resources are vital to an effective health care system. This variable i.e. to implement online heath care system using cloud computing was derived from two questions from the General health section of the surveys. The first question was framed as follows: -Traditionally to solve health related issues people were required to visit the hospitals personally, take an appointment, wait for the doctor to get free and then get the checkup done. After all this procedure the person has to again visit the hospital to collect his test reports. All this task was a great hectic and time consuming. The second question was framed as:-The person was unable to gain the information regarding approximate amount of the particular test, charges of the hospital. Taking in consideration all the above problems we are designing a system which would prove useful to the people and would save them from all the hectic procedures. 


\section{EXISTING SYSTEM}

\section{A. Thyrocare Android Application:-}

This application is designed only for thyroid related patients. We can only gain the information about thyroid. By using this application one can get the idea only related to specific thyroid test.

\section{B. E-Wireless Health Care System:-}

This system is designed to provide information regarding only a particular hospital. By using this application it is possible to maintain details of doctors and patients for a specific hospital.

\section{Practo Android Application:-}

This application provides detailed information about the doctors and also the online appointment booking facility. The main drawback of this application is that online generation of the report does not take place.

\section{PROPOSED SYSTEM}

To overcome all the drawbacks of the existing systems, online healthcare system using the concept of cloud computing have been proposed. This system will be helpful for dealing with all kinds of health related issues. An integrated system to have your health checkups done quickly at best price via nearest path labs and access your reports anywhere anytime on your phone. Based on reports get symptoms and preventive measures on your phone. If in doubts you can contact specialized doctors in your locality from your phone. If you ever need blood its just tap away. Application will broadcast your need to people with same blood group in your locality (city) so that if rare blood groups it will be savior for you and will save your blood bank expenses. For finding the location of the specific user Geolocation API is used which will help to identify residual end points i.e. latitude and longitude of the area. Integrating android application and cloud computing concepts can resolve many health issues and help user to perform many tasks like health tests, blood donation and obtaining preventive measures, symptoms of any disease.

\section{System Model}

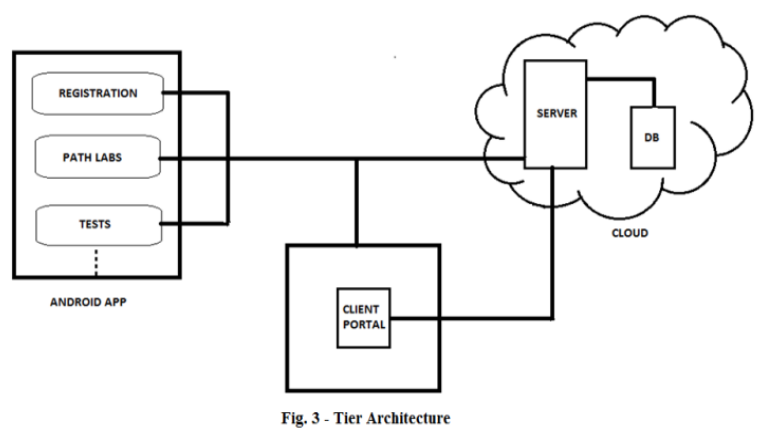

3-tier architecture includes mainly 3 components:

\section{a. Android application:}

This component is used to maintain feasibility of the user. By using this component user can able to easily access the data related to the path labs, hospitals, tests, offers, etc. It provides user interface and increases the portability of the user.

\section{b. Cloud:}

This is the second component of three tier architecture. It includes server and Database and handles them in cloud. Server plays the main role in the overall system as the whole authentication is done by server only and it also include the whole database.

c. Client portal:

It is the third component of three tier architecture. As the system is able to provide the pathlabs then the client portal helps to register themselves with the system. The flow of the system is given in Fig.2

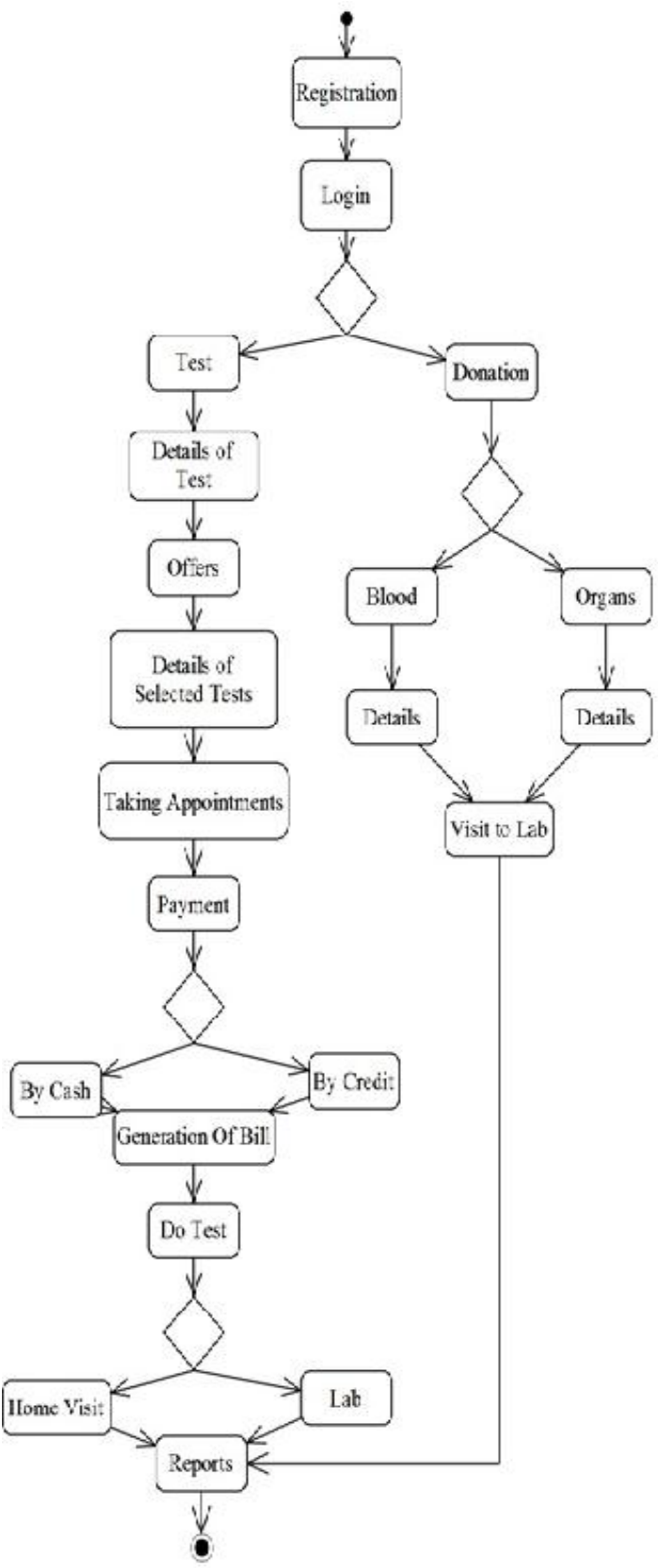

Fig.2 Flow of the System

\section{ADVANTAGES}

1. Easy access to path labs at competitive price. 2. Easy and any time access to your reports. 3. Symptoms and preventive measures according to reports. 


\section{VII.OBJECTIVE}

1. Connect common people with different path labs in his/her locality.

2. Provide information about preventive measures and tips depending upon user's health check up results.

3. If user needs to broadcast any critical medical urgency to rest of users, he shall be able to do so. E.g. Bombay blood group requirement.

4. Act as central repository for user's all check up reports.

\section{VIII.SCOPE}

1. The project provides online platform for health check up bookings with cheaper rates.

2. It provides online repository for user's health reports and medical history.

3. It provides information about preventive measures and tips to user based on his/her Medical history.

\section{APPLICATION}

1. User can see path labs in his locality.

2. Book online health checkups appointments at competitive prize.

3. User can access checkups reports on application.

4. User can see symptoms and preventive measures depending on his reports.

5. User can book doctor's appointments.

6. Broadcast blood requirements.

\section{CONCLUSION}

A health system, also sometimes referred to as health care system or healthcare system is the organization of people, institutions, and resources that deliver health care services to meet the health needs of target populations. For health checkups there are many Systems available such as thyrocare android app, Ewireless healthcare system, practo etc. So taking in mind these apps were efficient for only specific health related issues. Thyrocare android app is only helpful for thyroid affected persons. E-wireless healthcare system is only helpful for maintaining data about particular hospital. Practo app is helpful for just obtaining the detailed information of doctors in our locality and taking online appointments.

Hence to overcome all the drawbacks of the existing systems we are introducing and implementing the online healthcare system using cloud computing concepts which will be efficient for providing online health checkups booking facilities with discounted rates, to get information about preventive measures and tips to avoid contagious diseases, provide different path labs and System will be able to keep track of user's health checkups records, if in case anybody requires the blood then he can broadcast his requirement on the app directly and notification will be send to all the users who are registered having the same blood group via messages and emails.

Thus our proposed system will be helpful in avoiding lots of hectic work such as:

1. Paper work

2. Time

3. In emergency cases for Blood

4. Finding nearest path labs, hospital

\section{REFERENCES}

[1] Saldarriaga, A, J., Pérez, J.J. Restrepo, J., and Bustamante, J." A mobile application for ambulatory electrocardiographic monitoring inclinical and domestic environments", IEEE Health Care Exchanges (PAHCE), 2013 Pan American, and pp: 1-4, 2013.

[2] Doukas, C., and Maglogiannis, I., " Managing Wearable Sensor Data through Cloud Computing", Third IEEE International Conference on Cloud Computing Technology and Science (CloudCom), pp: 440-445, 2011.

[3] Ibnualim, I., and Supangkat, S, H., "Design of Health Social Media to Improve the Quality of Patient's Recovery", International conference on Cloud Computing and Social Networking (ICCCSN), pp: 1-4, 2012.

[4] Ratnam, K, A., and Dominic, P, D, D.," Cloud Services Enhancing the Malaysian Healthcare Sector", international conference on Computer \& Information Science (ICCIS), Vol: 2, pp: 604-608, 2012.

[5] Deng, M., Petkovic, M., Nalin, M., and Baroni, I.," A home healthcare system in the cloud-addressing security and privacy challenges", IEEE International Conference on Cloud Computing (CLOUD), pp: 549-556, 2011.

[6] Pirahandeh, M., Kim, D., " Co-Designing an Intelligent DoctorsColleagues-Patients Social Network", International Conference on Cloud Computing and Social Networking (ICCCSN), pp: 1-4, 2012.

[7] R. Zhang, and L. Liu L. Security models and requirements for healthcare application clouds. In Proceedings of $3 \mathrm{rd}$ International Conference on Cloud Computing 2010. IEEE Cloud '10, Miami, Florida, USA, 268-275, 2010.

[8] Chao-Tung Yang; Lung-Teng Chen; Wei-Li Chou; Kuan-Chieh Wang; , "Implementation of a Medical Image File Accessing System on Cloud Computing," Computational Science and Engineering (CSE), 2010 IEEE 13th International Conference on ,vol., no., pp.321-326, 11-13 Dec. 2010.

[9] $Y u, D$. Weider D. R. Bhagwat, "Modeling Emergency and Telemedicine Heath Support System: A Service Oriented Architecture Approach Using Cloud Computing.." IJEHMC 2 , no.3 (2011): 63-88, 2011

[10] V, Koufi, F. Malamateniou, G. Vassilacopoulos, "Ubiquitous access to cloud emergency medical services," Information Technology and Applications in Biomedicine (ITAB), $201010^{\text {th }}$ 